\title{
Cross-modal sensory transfer: Bees do it
}

\section{Insert Deck Here}

\author{
By Gerhard von der Emde ${ }^{1}$ and Theresa Burt de Perera ${ }^{2}$
}

tion that can be accessed by multiple senses. Up to now, spontaneous cross-modal object recognition has only been described in mammalian species (humans, apes, monkeys, dolphins, and rats) (6-8) and in one aquatic creature-the weakly electric elephantnose fish (9).

In an elegant experiment, Perry et al. trained bumblebees to discriminate two differently shaped objects (cubes and spheres) using only touch (in darkness) or only vision (in light, but barred from touching the objects). After training, the bumblebees discriminated between the same objects using only the other sensory information. This shows that, the brain encoded the sensory input in a way that allowed the two sensory channels to exchange information and compare and match objectrelated inputs.

The mechanisms by which bees achieve this task have yet to be elucidated. Perhaps the bee's brain stores a representation of the object in a way that allows it to be accessed and understood across different sensory systems. The sensory information would need to be integrated to form a representation that is independent of the sensory modality through which the information was introduced (that is, both senses use a matched encoding format). This would enable bees to recognize objects cross-modally without any previous experience, and cross-modal object recognition would not require training. Alternatively, information that originates from multiple senses might initially be unmatched in format, and cross-modal object recognition could depend on sensory experience and learning. In this case, bees might have learned to associate visual and tactile inputs of basic features common in other environmental objects when exposed to these features in the past. For example, a bee might have learned to associate a visual and a tactile image of a curved edge or a corner. Subsequently, these associations would generalize to new objects and new situations.

Whatever the mechanism, cross-modal object recognition requires storage, in the brain, of a representation of the object's features that can be accessed by different senses. This would imply that an object can be recognized with a sense through which it had never been experienced, before. Perry et al. show that this cognitive capability can be achieved by the brain of an insect, which contains just a small fraction of the number of neurons in a vertebrate brain (10).

How the organization of sensory systems achieves cross-modal object recognition is a key unanswered question. Sense organs respond to the physical stimuli of the outside world and transduce them into a series of action potentials encoding the specific stimulus parameters. Connections are then made with nerve cells in dedicated sensory nuclei within the brain, which in turn project to subsequent sensory nuclei for further processing (see the figure). To add to the complexity, these following centers also project back to lower processing centers, forming reciprocal circuits. For multisensory integration and cross-modal transfer, information from the different sensory modalities that encode characteristics of the same object must come together, eventually forming a multi-sensory representation of the object. In most cases, scientists still do not know exactly where in the sensory pathway this occurs, particularly in bees and fish, which do not possess specialized cortical structures. One possibility is that higher brain structures, such as the cortex in mammals or the mushroom bodies and central complex in bees, form the anatomical substrate for multisensory representations $(6,11)$. Alternatively, because crosstalk between the senses also occurs at lower levels (e.g., in the midbrain and diencephalon) $(1,12,13)$ and sensory nuclei on different levels are connected in a reciprocal manner, a distributed representation of object properties might occur in several interconnected multi-sensory nuclei (see the figure).

The fact that bees can achieve cross-modal object recognition might have implications for how we think about cognition in general. In humans, scientists assume that this ability involves mental imagery (14) based on internal representations in higher brain centers. Thus it has been argued that this task relies on awareness. Whether that is also the case in bumblebees is a matter of debate as simpler explanations are possible. Whatever the underlying mechanism, the newly found ability of bumblebees to perform cross-modal recognition shows that, like humans, they possess a sensory integration system that allows them to form a complex representation of their world.

REFERENCES AND NOTES
1 Institute of Zoology, University of Bonn, Bonn

2 Department of Zoology, University of Oxford, U.K. E-mail: theresa.burt@sjc.ox.ac.uk 
1.B. E. Stein, T. R. Stanford, B. A. Rowland, J.Neurosci. 40, 3 (2020).

2. M. O. Ernst, M. S. Banks, Nature 415, 429 (2002). 3.C. J. Perry, S. Guttierrez Al-Khudhairy, L. Chittka, Science XX, XXX (2020).

4.J. Guo, A. Guo, Science 309, 307- (2005).

5.L. Proops, K. McComb, D. Reby, Proc. Natl. Acad. Sci. U.S.A. 106, 947 (2009)

6.B. D. Winters, J. M. Reid, J. Neurosci. 30, 6253 (2010).

7.L. M. Herman, A. A. Pack, M. Hoffmann-Kuhnt, J. Comp. Psychol. 112, 292 (1998).

8.A. Cowey, L. Weiskrantz, Neuropsychologia 13 117 (1975)

9.S. Schumacher, T. Burt de Perera, J. Thenert, G. von der Emde, Proc. Natl. Acad. Sci. U.S.A. 113 7638 (2016).

10.M. Giurfa, Trends Neurosci. 36, 285 (2013).

11.F. Peng, L. Chittka, Curr. Biol. 27, 224 (2017).

12.M. Zeymer, G. von der Emde, M. F. Wullimann, Frontiers Neuroanat. 12, 79 (2018).

13. H. F. Sperdin, C. Cappe, M. M. Murray, Frontiers Neurosci. 4, 9 (2010).

14.B. Nanay, Cortex 105, 125 (2018).

Acknodedgments:[AU: please add COH here if any]

10.1126/science.aba 8519

Figure 1: Schematic sketch for cross-modal object recognition representing general principles across animal groups.

In both vertebrates and invertebrates, sense organs (such as eyes for vision or fingertips - antenna in bumblebees - for touch) respond to physical stimuli in the outside world and transfer this information to specific sensory nuclei of the brain, which in turn are connected in a reciprocal manner with secondary nuclei. While sensory pathways start monomodally, in many cases multimodal neurons are found fairly early in the sensory pathway. In higher centers, multimodal sensory nuclei are the norm. In principle, crossmodal sensory transfer might occur at any stage past the first, monomodal sensory nuclei.

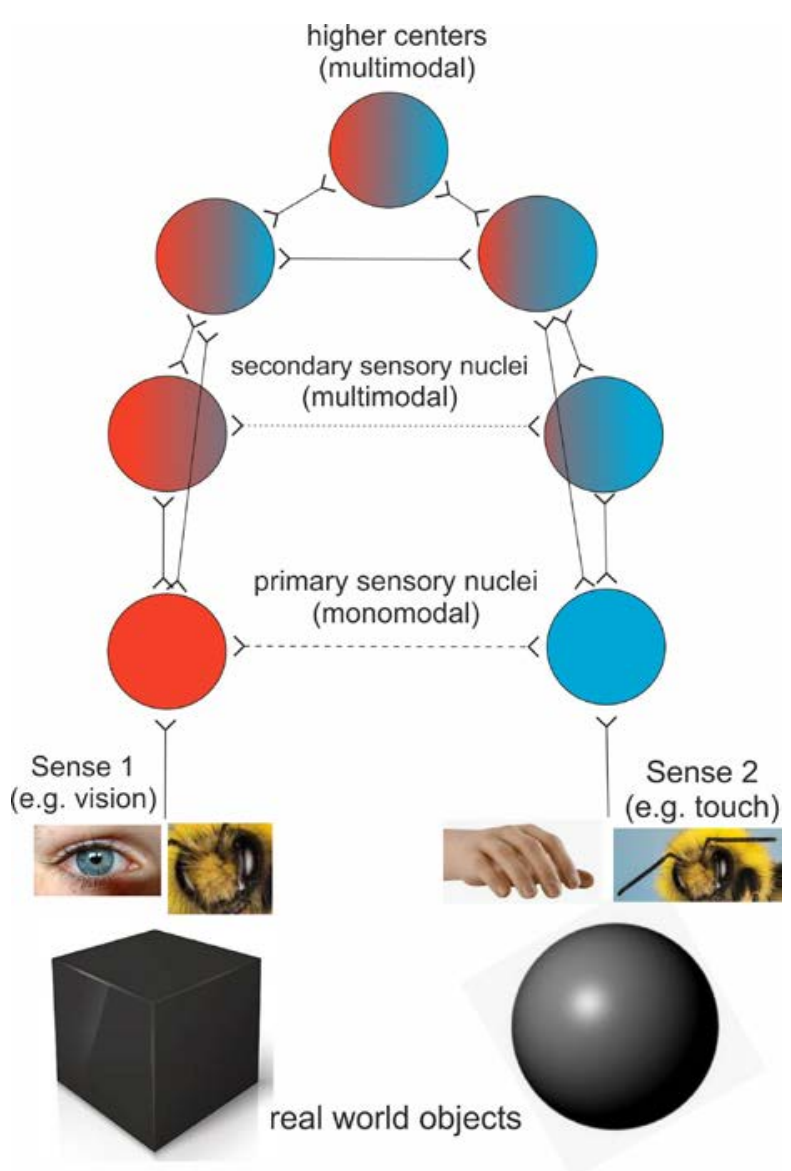

